\title{
Estado nutricional de escolares em Porto Velho, Rondônia'
}

\author{
Nutritional status of schoolchildren in \\ Porto Velho, Rondônia, Brazil
}

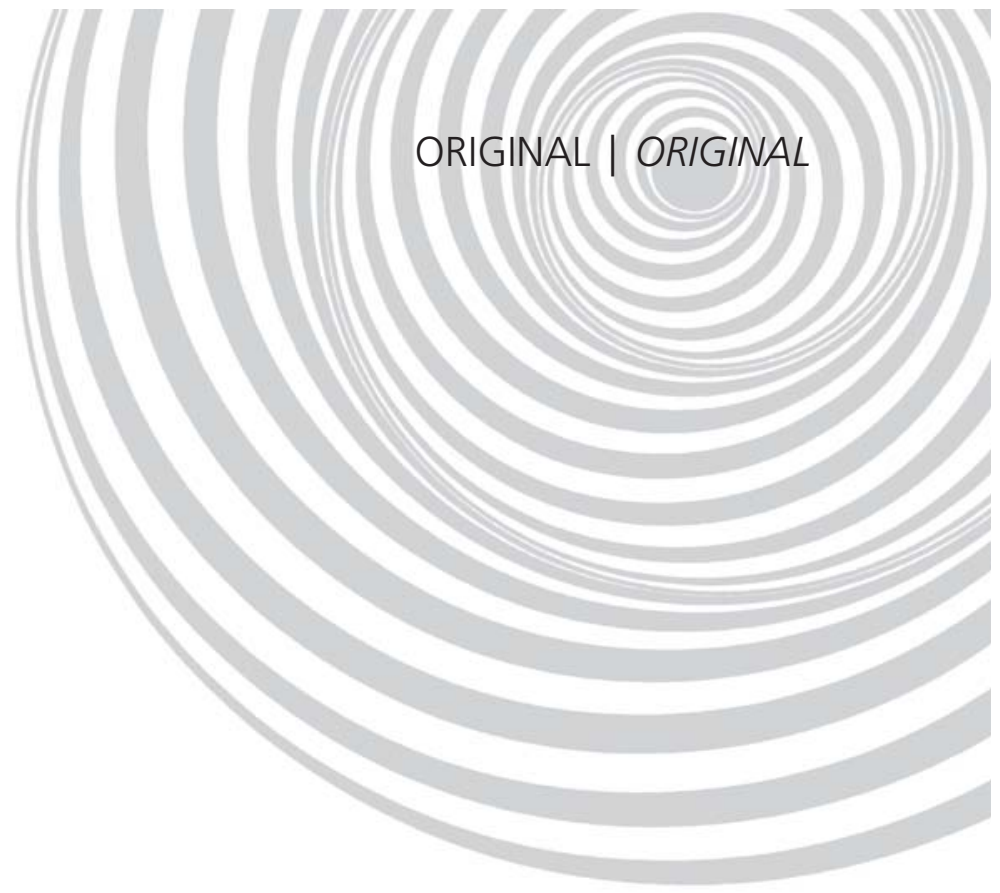

Edson dos Santos FARIAS 2,3

Gil GUERRA-JÚNIOR ${ }^{3,4}$

Édio Luiz PETROSKI ${ }^{5}$

RE S U M O

\section{Objetivo}

Avaliar o estado nutricional em escolares da rede municipal de ensino da cidade de Porto Velho (RO) em relação à idade, ao sexo e às características sócio-demográficas em uma população de baixo nível socioeconômico.

\section{Métodos}

A amostra constituiu-se de 1057 escolares com baixo nível socioeconômico, de ambos os sexos, entre 7 e 10 anos, selecionada por meio de amostragem aleatória proporcional por setor e, divisão intencional por conglomerado de turma. Foi utilizado um questionário para obter informações sobre os dados sóciodemográficos. Os escolares foram classificados segundo o peso para a estatura em desnutridos, eutróficos, sobrepesos e obesos; e segundo o peso para a estatura e estatura para idade em eutróficos, desnutridos agudos, pregressos ou crônicos.

\section{Resultados}

Não foi observada diferença significativa entre os sexos para peso ou estatura. Em relação apenas ao peso para a estatura, 86,0\% foram classificados com eutróficos, 4,0\% desnutridos, 7,0\% sobrepesos e 3,0\% obesos. Em relação aos índices de peso para estatura e estatura para idade, 89,0\% foram classificados em eutróficos, 2,5\% desnutridos agudos, 7,0\% desnutridos pregressos e 1,5\% desnutridos crônicos.

\footnotetext{
1 Artigo elaborado a partir da dissertação de E.S. FARIAS, intitulada "Estado nutricional, crescimento físico e atividade física de escolares de sete a dez anos de idade da rede municipal de ensino de Porto Velho, RO". Programa de Pós-Graduação em Educação Física, Universidade Federal de Santa Catarina; 2002.

2 Universidade Federal do Acre, Departamento de Educação Física e Desporto. Rio Branco, AC, Brasil.

${ }^{3}$ Universidade Estadual de Campinas, Faculdade de Ciências Médicas, Centro de Investigação em Pediatria, Laboratório de Crescimento e Composição Corporal. Cidade Universitária, Caixa Postal 6111, 13083-970, Campinas, SP, Brasil. Correspondência para/Correspondence to: G. GUERRA-JÚNIOR. E-mail: <gilguer@fcm.unicamp.br>.

${ }^{4}$ Universidade Estadual de Campinas, Faculdade de Ciências Médicas, Departamento de Pediatria. Campinas, SP, Brasil.

${ }^{5}$ Universidade Federal de Santa Catarina, Centro de Desportos, Departamento de Educação Física. Florianópolis, SC, Brasil.
} 
402 E.S. FARIAS et al.

\section{Conclusão}

Os escolares do ensino fundamental da rede pública municipal de Porto Velho (RO) com baixo nível socioeconômico apresentaram baixa prevalência de desnutrição ou sobrepeso/obesidade. Estes dados encontrados podem ser comparados aos de outras cidades e regiões do Brasil.

Termos de Indexação: Desnutrição. Estado nutricional. Fatores socioeconômicos. Obesidade. Saúde escolar.

\section{A B S T R A C T}

\section{Objective}

This study aimed to determine the nutritional status of schoolchildren in public schools in Porto Velho, Rondônia, Brazil, in relation to age, gender and sociodemographic features in a population of low socioeconomic status.

\section{Methods}

The sample comprised 1,057 schoolchildren of low socioeconomic status of both genders, aging from 7 to 10 years, selected by stratified random sampling by sector and purposeful sampling by group. Sociodemographic data were obtained with a questionnaire. The schoolchildren were classified according to their weight-for-height into malnourished, well nourished and overweight/obese and according to their weight-for-height and height-for-age into well nourished, severely malnourished, chronically malnourished or formerly malnourished.

\section{Results}

There was no significant difference between males and females for weight and height. In relation to weight-for-height alone, $86.0 \%$ were normal, 4.0\% were malnourished, $7.0 \%$ were overweight and 3.0\% were obese. In relation to weight-for-height and height-for-age, $89 \%$ were normal, $2.5 \%$ were acutely malnourished, $7.0 \%$ were formerly malnourished and 1.5\% were chronically malnourished.

\section{Conclusion}

The prevalence of malnutrition or overweight/obesity was low among elementary schoolchildren of low socioeconomic status in public schools of Porto Velho, Rondônia, Brazil. These data can be compared with data from other Brazilian cities and regions.

Indexing terms: Malnutrition, Nutritional status, Socioeconomic factors, Obesity, School health.

\section{N T R O D U ÇÃ O}

Nas últimas décadas vêm ocorrendo melhorias nas condições de saúde das crianças de quase todo o mundo, em especial no Brasil. A difusão das medidas de higiene e saúde pública tem propiciado importante queda na incidência de doenças infecciosas ${ }^{1}$. Por outro lado, a vida urbana nas sociedades modernas tem se associado às mudanças de comportamento, principalmente com relação à dieta e à atividade física, proporcionando aumento significativo das taxas de sobrepeso e obesidade ${ }^{2,3}$.

São fatores essenciais para a expressão completa da herança de crescimento, a alimentação e a atividade física, além da ausência de enfermidades orgânicas e psicológicas ${ }^{4}$. Assim, o monitoramento contínuo do crescimento e do estado nutricional de crianças é uma forma prática e de baixo custo, visto que as suas alterações podem ocorrer em qualquer faixa etária, desde os primeiros anos de vida 5 . Para as crianças em idade escolar, a vigilância nutricional serve como instrumento de avaliação de medidas de intervenção, como a merenda escolar6.

As avaliações do estado nutricional populacional, em especial de crianças, escolares ou não, são tradicionalmente executadas a partir da observação do peso, da estatura em relação à idade e ao sexo da criança ${ }^{7}$. No Brasil, há vários registros recentes de estudos sobre a avaliação do estado nutricional de crianças em escolas ou 
em comunidades, usando a antropometria como um indicador ou método direto, com atenção tanto para a desnutrição como para a obesidade $^{8-16}$.

A literatura médica mostra um grande número de estudos relacionando estado nutricional, em especial obesidade e sobrepeso, com nível socioeconômico, no entanto, Sobal \& Stunkard ${ }^{17}$, revisando 140 publicações, mostraram que existe uma associação inversa entre o nível socioeconômico e o excesso de peso apenas em mulheres de países desenvolvidos. Esta relação não é bem definida para homens e crianças. Em países em desenvolvimento, a obesidade parece estar associada ao maior nível socioeconômico $^{17-19}$

A avaliação nutricional em crianças e adolescentes de escolas municipais, na região Norte do Brasil, é pouco explorada na literatura. Assim sendo, o objetivo deste estudo foi avaliar o estado nutricional por idade, sexo e características sóciodemográficas em uma população de baixo nível socioeconômico do Ensino Fundamental I da cidade de Porto Velho (RO), Brasil.

\section{M É T O D O S}

Trata-se de um estudo descritivo e transversal, realizado em escolares do Ensino Fundamental I da rede municipal de Porto Velho (RO), no segundo semestre de 2001.

A população de escolares da rede municipal urbana de Porto Velho foi estimada, por meio do censo escolar de 2000, em 14897 alunos, distribuídos em 22 escolas. Desse número, 7263 estavam entre a $1^{\text {a }}$ e a $4^{\text {a }}$ séries do Ensino Fundamental I.

A população deste estudo foi constituída de escolares com idade compreendida entre 7 e 10 anos, de ambos os sexos, matriculados de $1^{\mathrm{a}}$ a $4^{\mathrm{a}}$ séries do Ensino Fundamental I, nos estabelecimentos da rede municipal de ensino da cidade de Porto Velho (RO).
O tamanho amostral foi calculado considerando-se o intervalo de confiança de $95 \%$ e uma margem de erro amostral tolerável de $3 \%$, utilizando a fórmula proposta por Barbetta ${ }^{20}$. Portanto, a amostra composta por 1057 escolares de 7 a 10 anos, de ambos os sexos, superou o tamanho amostral mínimo de 967 escolares.

Esta amostra obedeceu aos seguintes critérios: a) divisão, de forma intencional, da cidade em 4 setores ( $A$ a D: setor A com 7 escolas e os demais setores com 5 escolas cada), realizada pela localização geográfica das principais avenidas da cidade; b) dentro de cada setor, sorteio por processo aleatório de uma escola que atendia aos propósitos do estudo; c) escolha de todas as turmas de $1^{\mathrm{a}}$ a $4^{\mathrm{a}}$ séries na faixa etária de 7 a 10 anos de idade; d) o agrupamento das idades foi estabelecido pelos critérios estabelecidos por Ross \& Marfell-Jones ${ }^{21}$ em cada idade, utilizando os intervalos de $-0,50$ a $+0,49$ (ex: de 7,5 a 8,49 anos $=8$ anos).

O número de crianças que se recusou a participar da pesquisa foi sempre inferior a 10\% do total de alunos por turma.

O estudo foi aprovado pelo Comitê de Ética em Pesquisa com Seres Humanos da Universidade Federal de Santa Catarina (UFSC), com o protocolo número 131/01. A coleta de dados foi realizada mediante a autorização da Secretária Municipal de Educação de Porto Velho (RO). Após esclarecimento do trabalho junto à direção das escolas e aos pais das crianças, a coleta de dados foi realizada durante o segundo semestre de 2001. Todos receberam orientações sobre a pesquisa e a autorizaram. A coleta de todos os dados foi realizada unicamente pelo autor ESF, com o objetivo de obter as informações da forma mais padronizada.

Além dos dados de idade, sexo, local de nascimento (cidade e estado), com quem mora (ambos os pais, um dos pais, parentes ou outros), número de irmãos e número total de moradores na residência, o protocolo constou da avaliação do nível socioeconômico por meio de questionário padronizado pela Associação Nacional de Empresas de Pesquisa (ANEP) de 1997, com base 
404 E.S. FARIAS et al.

nos bens e utensílios domésticos e na escolaridade do chefe da família. O procedimento para aplicação do questionário social e demográfico foi realizado mediante uma reunião com os pais dos alunos, em dois momentos. No primeiro, com os pais de escolares de $1^{\text {a }}$ e $2^{\text {a }}$ séries; no segundo, com pais de escolares de $3^{a}$ e $4^{a}$ séries. A partir desses dados, as famílias foram classificadas em quatro níveis socioeconômicos ( $A=1$ família=0,1\%; $B=32=3,1 \% ; C=307=29,0 \% ; D=717$ $=67,8 \%)$. No grau de instrução do chefe da família, predominou o fundamental incompleto (554 casos $=52,4 \%$ ), seguido de fundamental completo $(214=20,4 \%)$, médio incompleto (134=12,7\%), médio completo $(108=10,2 \%)$, superior incompleto $(21=2,0 \%)$ e superior completo $(26=2,5 \%)$.

$\mathrm{Na}$ avaliação antropométrica foram medidos o peso (P) em kg, utilizando-se balança antropométrica mecânica Filizola ${ }^{\circledR}$, com escala de 100 gramas, e a estatura (E) em metros, por meio de um estadiômetro vertical tipo trena, com $2 \mathrm{~m}$, com uma precisão de $0,1 \mathrm{~cm}$. Os alunos foram pesados e medidos uma única vez, descalços e apenas com o uniforme da escola.

Com relação à avaliação do estado nutricional, foram utilizados os índices de peso para estatura (P/E) e de estatura para idade (E/I), expressos em unidades de desvio-padrão (Z-escore) em relação ao padrão de referência do National Center of Health Statistics ${ }^{22}$, calculado utilizandose o programa Nutrition do pacote Epi Info versão $2002^{23}$ do Centers for Disease Control and Prevention. Os pontos de corte adotados foram os Z-escores abaixo de -2,0 e acima de $+2,0$ da população de referência.

A partir da utilização dos valores de Z-escore para P/E, a classificação adotada foi: desnutrição $=z<-2,0$; eutrofia $=-2,0 \leq z \leq+1,0$; sobrepeso $=+1,0<z \leq+2,0$; e obesidade $=z>+2,0$. Quando utilizados z escore dos índices de P/E e E/I, a classificação nutricional adotada foi a adaptação da proposta por Waterlow et al. ${ }^{24}$, em que eutrofia: $P / E$ e E/I com z> -2,0; desnutrição aguda: $P / E \leq-2,0$ e $E / I>-2,0$; desnutrição pregressa: $P / E>-2,0$ e E/I $\leq-2,0$; e desnutrição crônica: $P / E$ e $E / I \leq-2,0$.
Para a tabulação e a análise estatística dos dados foram utilizados o programa SPSS for Windows versão 11.0 e o programa Epi Info (2002), para avaliação e classificação do estado nutricional. Foi realizada análise descritiva tendo sido aplicados os testes de Qui-quadrado e de Fisher para verificar diferenças no estado nutricional em relação ao sexo, à idade e ao número de pessoas que moram na mesma casa, e o teste de Mann-Whitney para verificar diferenças em relação ao sexo para $\mathrm{P}$ e E em valores absolutos nas diferentes idades. O nível de significância adotado foi de $5 \%$.

\section{RES U LT A DOS}

Entre os 1057 escolares avaliados houve um predomínio de meninas $(51,4 \%)$, com idade de 10 anos (33,9\%), nascidas em Porto Velho

Tabela 1. Características sociais e demográficas de 1057 escolares da rede municipal de Porto Velho (RO), 2002.

\begin{tabular}{|c|c|c|}
\hline Variável & $n$ & $\%$ \\
\hline \multicolumn{3}{|l|}{ Sexo } \\
\hline Masculino & 514 & 48,6 \\
\hline Feminino & 543 & 51,4 \\
\hline \multicolumn{3}{|l|}{ Idade } \\
\hline 7 anos & 142 & 13,4 \\
\hline 8 anos & 265 & 25,1 \\
\hline 9 anos & 292 & 27,6 \\
\hline 10 anos & 358 & 33,9 \\
\hline \multicolumn{3}{|l|}{ Local de nascimento } \\
\hline Porto Velho & 872 & 82,5 \\
\hline Outras localidades & 185 & 17,5 \\
\hline \multicolumn{3}{|l|}{ Com quem mora } \\
\hline Pai e mãe & 658 & 62,3 \\
\hline Pai & 65 & 6,1 \\
\hline Mãe & 276 & 26,1 \\
\hline Parentes & 56 & 5,3 \\
\hline Outros & 02 & 0,2 \\
\hline \multicolumn{3}{|l|}{ Número de irmãos } \\
\hline Nenhum & 86 & 8,1 \\
\hline $1-2$ & 557 & 52,7 \\
\hline $3-4$ & 300 & 28,4 \\
\hline $5-6$ & 97 & 9,2 \\
\hline$>6$ & 17 & 1,6 \\
\hline \multicolumn{3}{|c|}{ Número de pessoas que moram na mesma residência } \\
\hline Até 2 & 20 & 1,9 \\
\hline $3-4$ & 345 & 32,6 \\
\hline $5-6$ & 519 & 49,1 \\
\hline$>6$ & 173 & 16,4 \\
\hline
\end{tabular}


(82,5\%), que moram com ambos os pais (62,3\%), que têm um ou dois irmãos $(52,7 \%)$ e que têm cinco ou seis pessoas morando na mesma residência (49,1\%), conforme a Tabela 1.

Não foi observada diferença estatisticamente significante entre os sexos nas diferentes faixas etárias para P e E (Tabela 2).

Segundo a distribuição dos dados em Z-escore dos índices de E/I e P/E, 940 escolares $(89,0 \%)$ foram classificados como eutróficos, 25
(2,5\%) como desnutridos agudos, 75 (7,0\%) como desnutridos pregressos e 17 (1,5\%) como desnutridos crônicos. Em relação à idade e ao sexo, somente foi observada diferença estatisticamente significante no sexo quando comparados os desnutridos crônicos em relação aos eutróficos, com predomínio de desnutrição crônica nas meninas (Tabela 3).

Ao considerar apenas os dados em Z-escore do P/E, 910 (86\%) foram classificados como

Tabela 2. Dados de média (M) e desvio-padrão (DP) de peso e de estatura em relação à idade e ao sexo de 1057 escolares da rede municipal de Porto Velho (RO), 2002.

\begin{tabular}{|c|c|c|c|c|c|c|c|c|c|c|}
\hline \multirow[b]{2}{*}{ Idade (anos) } & \multicolumn{5}{|c|}{ Masculino } & \multicolumn{5}{|c|}{ Feminino } \\
\hline & $n$ & $M$ & DP & $M$ & DP & $n$ & $M$ & DP & $M$ & DP \\
\hline 8 & 129 & 24,6 & 4,1 & 124,5 & 5,8 & 136 & 24,2 & 4,1 & 124,7 & 6,1 \\
\hline 9 & 137 & 27,7 & 6,0 & 130,0 & 5,5 & 155 & 27,3 & 5,5 & 130,0 & 6,4 \\
\hline
\end{tabular}

Teste de Mann-Whitney - sexo masculino x feminino: valores de $p$; 7 anos: Peso: 0,795; Estatura: 0,282; 8 anos: Peso: 0,361; Estatura: 0,560; 9 anos: Peso: 0,570; Estatura: 0,818; 10 anos: Peso: 0,343; Estatura: 0,279.

Tabela 3. Dados da avaliação nutricional dos escolares de Porto Velho (RO), de acordo com o critério de Waterlow et al. ${ }^{24}$ adaptado em relação à idade e ao sexo. 2002.

\begin{tabular}{|c|c|c|c|c|c|c|c|c|}
\hline \multirow{2}{*}{$\begin{array}{l}\text { Idade } \\
\text { (anos) }\end{array}$} & \multicolumn{2}{|c|}{ Desnutrição crônica } & \multicolumn{2}{|c|}{ Desnutrição pregressa } & \multicolumn{2}{|c|}{ Desnutrição aguda } & \multicolumn{2}{|c|}{ Eutrofia } \\
\hline & Masculino & Feminino & Masculino & Feminino & Masculino & Feminino & Masculino & Feminino \\
\hline 7 & 0 & 0 & 2 & 6 & 3 & 3 & 69 & 59 \\
\hline 8 & 1 & 3 & 16 & 12 & 3 & 2 & 109 & 119 \\
\hline 9 & 0 & 0 & 12 & 7 & 5 & 5 & 120 & 143 \\
\hline 10 & 2 & 11 & 9 & 11 & 1 & 3 & 162 & 159 \\
\hline Total & 3 & 14 & 39 & 36 & 12 & 13 & 460 & 480 \\
\hline
\end{tabular}

$\chi^{2}$ Eutrofia $x$ tipos de desnutrição em relação ao sexo; Crônica: $\chi^{2}=6,55 ; p=0,010$; Pregressa: $\chi^{2}=0,26 ; p=0,609 ;$ Aguda: $\chi^{2}=0,01 ; p=0,926$.

Tabela 4. Dados da avaliação nutricional dos escolares de Porto Velho (RO), de acordo com o critério de peso por estatura em relação à idade e ao sexo. 2002.

\begin{tabular}{|c|c|c|c|c|c|c|c|c|}
\hline $\begin{array}{l}\text { Idade } \\
\text { (anos) }\end{array}$ & \multicolumn{2}{|c|}{ Desnutrição } & \multicolumn{2}{|c|}{ Eutrofia } & \multicolumn{2}{|c|}{ Sobrepeso } & \multicolumn{2}{|c|}{ Obesidade } \\
\hline 7 & 3 & 3 & 65 & 57 & 4 & 6 & 2 & 2 \\
\hline 8 & 4 & 5 & 107 & 117 & 15 & 10 & 3 & 4 \\
\hline 9 & 5 & 5 & 116 & 137 & 9 & 9 & 7 & 4 \\
\hline 10 & 3 & 14 & 155 & 156 & 9 & 9 & 7 & 5 \\
\hline
\end{tabular}

$\chi^{2}$ Eutrofia $x$ desnutrição, sobrepeso e obesidade em relação ao sexo; Desnutrição: $\chi^{2}=2,70$; $p=0,100$; Sobrepeso: $\chi^{2}=0,31 ; p=0,577 ;$ Obesidade: $\chi^{2}=0,68 ; p=0,409$. 
406 | E.S. FARIAS et al.

Tabela 5. Dados da avaliação nutricional segundo peso por estatura de escolares de Porto Velho (RO), em relação ao número de pessoas que residem na mesma residência, 2002.

\begin{tabular}{|c|c|c|c|c|c|c|c|c|}
\hline \multirow{2}{*}{$\begin{array}{l}\text { Pessoas por } \\
\text { residência }\end{array}$} & \multicolumn{2}{|c|}{ Desnutrição } & \multicolumn{2}{|c|}{ Eutrofia } & \multicolumn{2}{|c|}{ Sobrepeso } & \multicolumn{2}{|c|}{ Obesidade } \\
\hline & $n$ & $\%$ & $n$ & $\%$ & $n$ & $\%$ & $n$ & $\%$ \\
\hline$\leq 2$ & 4 & 9,5 & 12 & 1,5 & 3 & 4,0 & 1 & 3,0 \\
\hline 3 a 4 & 12 & 28,5 & 281 & 31,0 & 33 & 46,5 & 19 & 56,0 \\
\hline$\geq 5$ & 26 & 62,0 & 617 & 68,5 & 35 & 49,5 & 14 & 41,0 \\
\hline
\end{tabular}

Eutrofia x Desnutrição e Eutrofia x Sobrepeso + Obesidade; em relação ao número de pessoas por residência; Desnutrição: $\chi^{2}{ }_{(2)}=16,36 ; p<0,001$; Sobrepeso + Obesidade: $\chi_{(2)}^{2}=84,54 ; p<0,001$.

eutróficos, 42 (4\%) como desnutridos, 71 (7\%) como sobrepesos e 34 (3\%) como obesos. Não foi observada diferença estatisticamente significante em relação ao sexo, quando comparados os desnutridos, sobrepesos e obesos em relação aos eutróficos (Tabela 4).

No entanto, quando a variável avaliada foi o número de pessoas que moram na mesma residência, foram observadas diferenças estatisticamente significantes, quando comparados os eutróficos em relação aos desnutridos, com predomínio dos desnutridos nas residências com menor número de pessoas; e, no caso dos sobrepesos e obesos, também houve diferença estatisticamente significante em relação aos eutróficos, com predomínio dos sobrepesos e obesos em residências com número intermediário (entre 3 e 4) de pessoas (Tabela 5).

\section{I S C U S S Ã O}

Apesar da existência na literatura de vários trabalhos a respeito do estado nutricional em escolares brasileiros, nenhum deles, com o mesmo perfil do presente estudo, foi realizado na região Norte do Brasil. Portanto, seus resultados serão comparados com dados de literatura em especial das regiões Sul, Sudeste e Nordeste do Brasil, onde se concentra a maioria dos estudos realizados.

As características deste estudo mostram que, entre os 1057 escolares avaliados, houve um predomínio de meninas, com idade de 10 anos, nascidas em Porto Velho, que moram com ambos os pais, que têm um ou dois irmãos, que têm cinco ou seis pessoas morando na mesma resi- dência, de famílias com nível socioeconômico D e com grau de instrução do chefe da família de Ensino Fundamental Incompleto. Estes dados caracterizam, sob todos os aspectos, uma população de nível socioeconômico muito baixo dentro de uma região pobre do Brasil.

Não foi encontrada diferença nos valores absolutos de $\mathrm{P}$ e $\mathrm{E}$ nas diversas faixas etárias em relação ao sexo, o que é estranho, pois no intervalo de idade utilizado no estudo dever-se-ia encontrar um grupo de meninas em puberdade e, portanto, com dados antropométricos superiores aos meninos da mesma faixa etária. O fato de não se encontrar esta diferença, e tendo-se em vista que o desenvolvimento puberal não foi avaliado, pode sugerir que a puberdade nesta região do Brasil esteja ocorrendo mais tardiamente ou que apenas a avaliação de $P$ e a $E$ não seja eficiente para demonstrar as alterações de composição corporal peri-puberdade. Anjos ${ }^{25}$, no Rio de Janeiro (RJ), avaliando outras variáveis de composição corporal como índice de massa corporal (IMC), dobras cutâneas e percentual de gordura corporal, conseguiu mostrar predomínio de gordura subcutânea nas meninas em relação aos meninos em todas as faixas etárias. Também no Rio de Janeiro (RJ), Chiara et al. ${ }^{26}$ mostraram que a gordura detectada pela dobra subescapular (duas a três vezes acima do esperado) encontrada em adolescentes, provavelmente tenha sido decorrente do excesso de acúmulo de tecido adiposo antes da adolescência. No entanto, Rolland-Cachera et al. ${ }^{27}$, realizando estudo com crianças, adolescentes e adultos jovens franceses (1 mês a 21 anos), não observaram diferenças na distribuição de gordura 
corporal entre os sexos e as idades. Talvez esses dados mostrem que fatores como a nutrição, o ambiente e a herança genética possam estar interferindo no resultado, quando confrontados com os dados do presente estudo, principalmente sabendo-se que as crianças avaliadas neste estudo apresentam características típicas desta região brasileira, diferindo em relação ao hábito alimentar, à etnia, à cultura, entre tantos outros aspectos.

Ao adaptar para a aplicação do Z-escore o método preconizado por Waterlow et al. ${ }^{24}$, foi possível identificar $2,5 \%$ das crianças como desnutridas agudas e 1,5\% como crônicas, sem diferenças de distribuição entre os sexos e as idades de 7 a 10 anos. Os estudos realizados no Brasil, utilizando esta mesma metodologia, são escassos. Anjos $^{25}$, avaliando 145 escolares de baixa renda em Nova Iguaçu (RJ), encontrou uma prevalência de $3,5 \%$ de desnutrição aguda e $6,2 \%$ de desnutrição crônica. Gross et al. ${ }^{28}$, avaliando crianças até 2 anos de uma favela da cidade do Rio de Janeiro, encontraram $4,9 \%$ de desnutridos agudos e 6,4\% de desnutridos crônicos, e Victora et al. ${ }^{29}$, avaliando 5 mil crianças do nascimento a 52 meses de idade em Pelotas (RS), encontraram dados entre $0,3 \%$ e 1,3\% de desnutrição aguda e 5,3\% a 12,2\% de desnutrição crônica. Esses dados, apesar da diferença das faixas etárias avaliadas, são semelhantes aos de Anjos ${ }^{25}$ e todos diferentes e muito maiores que os do presente estudo. No entanto, deve-se levar em consideração o lapso de tempo entre os dois estudos de cerca de 15 anos, sendo que neste período houve modificações significativas da tendência secular de desnutrição e obesidade em todas as regiões do Brasil; dados comprovados pela análise dos inquéritos nacionais realizados em 1974/75, 1989 e 1996"11.

Se, em um passado recente, o baixo nível socioeconômico esteve associado à desnutrição, vive-se no País, atualmente, uma transição nutricional em que o nível de escolaridade materna está intimamente associado à obesidade infantil. Taddei $^{30}$, analisando dados nacionais de 1989 e 1996, verificou aumento na obesidade entre filhos de mães com mais de quatro anos de escolaridade na região Nordeste, enquanto que essa tendência se invertia na região Sul, mostrando no País fenômenos distintos, em função do nível de desenvolvimento de cada região.

Ao utilizar apenas o dado de P/E foi possível identificar, neste estudo, uma prevalência de 4,0\% de desnutridos, $86,0 \%$ eutróficos, 7,0\% sobrepesos e 3,0\% obesos, sem diferença significante em relação ao sexo, quando comparados os desnutridos, sobrepesos e obesos em relação aos eutróficos. Os estudos de Monteiro \& Conde ${ }^{11}$, Silva et al. ${ }^{13}$ e Sotelo et al. ${ }^{15}$ adotaram o mesmo critério que o do presente estudo para avaliação da prevalência de sobrepeso e obesidade. Monteiro \& Conde ${ }^{11}$, avaliando os inquéritos nacionais realizados com crianças de zero a cinco anos da cidade de São Paulo, encontraram uma prevalência de $3,2 \%$ de obesidade no inquérito nacional de $1974 / 75,4,0 \%$ no de $1984 / 85$ e $3,8 \%$ no de 1995/96. Silva et al. ${ }^{13}$, avaliando crianças entre dois e cinco anos de escolas privadas de Recife (PE), encontraram $22,6 \%$ de sobrepeso e $11,3 \%$ de obesidade. Sotelo et al. ${ }^{15}$, estudando 2519 crianças entre seis e nove anos de idade de oito escolas públicas estaduais da Vila Mariana na cidade de São Paulo, observaram no sexo masculino $11,9 \%$ de sobrepeso e $10,3 \%$ de obesidade, e no sexo feminino, $13,7 \%$ e $11,7 \%$, respectivamente. Todos esses dados ultrapassam os observados no presente estudo, provavelmente devido às grandes diferenças socioeconômicas entre os grupos analisados nos diferentes estudos, como mostrado por Monteiro \& Conde ${ }^{11}$ e Silva et al. ${ }^{13}$, entre tantos outros estudos.

No presente estudo observou-se, em relação ao indicador P/E uma associação significante entre o número de moradores na residência e o aumento dos casos de desnutrição e de sobrepeso e obesidade. Quanto ao sobrepeso e à obesidade, a associação observada foi inversa. Pelto et al. ${ }^{31}$ observaram que quanto maior o tamanho da família, maior a possibilidade de encontrar crianças com baixo peso e baixa estatura para a idade. Essa associação também foi observada por 
Guimarães et al. ${ }^{32}$. Magalhães \& Mendonça ${ }^{33}$, em um estudo com adolescentes das regiões Sudeste e Nordeste, mostraram que morar em domicílio com até quatro pessoas se associou a sobrepeso e obesidade somente entre os meninos da região Nordeste; fato este sem explicação pelos autores.

Portanto, pode-se concluir que os escolares de baixo nível socioeconômico do Ensino Fundamental da rede municipal de Porto Velho (RO) apresentam baixos índices de desnutrição ou sobrepeso/obesidade, que podem ser comparados aos encontrados em outras cidades e regiões do Brasil.

\section{COLABORADORES}

E.S. FARIAS participou da elaboração do estudo, da coleta, da análise dos dados e da redação do artigo. G. GUERRA-JUNIOR participou da análise dos dados e da redação do artigo. E.L. PETROSKI participou da elaboração do estudo, da análise dos dados e da redação do artigo.

\section{REFERÊ N CIAS}

1. Pan-American Health Organization. Health statistics in the Americas. Washington (DC): Scientific Publications; 1995.

2. Popkin BM, Paeratakul S, Fengying Z, Keyou G. A review of dietary and environmental correlates of obesity with emphasis on developing countries. Obes Res. 1995; 3(Suppl. 2):135-43.

3. Pinheiro ARO, Freitas SFT, Corso ACT. Uma abordagem epidemiológica da obesidade. Rev Nutr. 2004; 17(4):523-33.

4. Marcondes E. Desenvolvimento da criança. Rio de Janeiro: Sociedade Brasileira de Pediatria; 1994.

5. Barros AA, Barros MB, Maude GH, Ross DA, Davies PS, Preece MA. Evaluation of the nutritional status of 1 st-year school children in Campinas, Brasil. Ann Trop Paediatr. 1990; 10(1):75-84.

6. Batista Filho M. Saúde e Nutrição. In: Almeida Filho $\mathrm{N}$, Rouquayrol $\mathrm{MZ}$, editores. Epidemiologia \& Saúde. Rio de Janeiro: Medsi; 1994.

7. Vasconcelos FAG. Avaliação nutricional de coletividades. Florianópolis: Editora da UFSC; 2000.

8. Ferreira HS, Albuquerque MIM, Ataíde TR. Estado nutricional de crianças menores de dez anos residentes em invasão do "Movimento dos SemTerra", Porto Calvo, Alagoas. Cad Saúde Pública. 1997; 13(1):137-9.

9. Ribas DLB, Philippi S, Tanaka AC, Zorzatto JR. Saúde e estado nutricional infantil de uma população da Região Centro-Oeste do Brasil. Rev Saúde Pública. 1999; 33(4):358-65.

10. Carvalho AT, Costa MJC, Ferreira LOC, Batista Filho M. Cartografia do retardo estatural em escolares do estado da Paraíba, Brasil. Rev Saúde Pública. 2000; 34(1):3-8.

11. Monteiro CA, Conde WL. Tendência secular da desnutrição e da obesidade na infância na cidade de São Paulo (1974 - 1996). Rev Saúde Pública. 2000; 34(6):52-61.

12. Leão LSCS, Araújo LMB, Moraes LTLP, Assis AM. Prevalência de obesidade em escolares de Salvador, Bahia. Arq Bras Endocrinol Metab. 2003; 47(2): 151-7.

13. Silva GAP, Balaban G, Freitas MMV, Baracho JDS, Nascimento EMM. Prevalência de sobrepeso e obesidade em crianças pré-escolares matriculadas em duas escolas particulares de Recife, Pernambuco. Rev Bras Saúde Mater Infant. 2003; 3(3):323-7.

14. Soar C, Vasconcelos FAG, Assis MAA, Grosseman $S$, Luna MEP. Prevalência de sobrepeso e obesidade em escolares de uma escola pública de Florianópolis, Santa Catarina. Rev Bras Saúde Mater Infant. 2004; 4(4):391-7.

15. Sotelo YOM, Colugnati FAB, Taddei JAAC. Prevalência de sobrepeso e obesidade entre escolares da rede pública segundo três critérios de diagnóstico antropométrico. Cad Saúde Pública. 2004; 20(1):233-40.

16. Silva GAP, Balaban G, Motta MEFA. Prevalência de sobrepeso e obesidade em crianças e adolescentes de diferentes condições socioeconômicas. Rev Bras Saúde Mater Infant. 2005; 5(1):53-9.

17. Sobal J, Stunkard AJ. Socioeconomic status and obesity: a review of the literature. Psychol Bull. 1989; 105(2):260-75.

18. Campos LA, Leite AJM, Almeida PC. Nível socioeconômico e sua influência sobre a prevalência de sobrepeso e obesidade em escolares do município de Fortaleza. Rev Nutr. 2006; 19(5):531-8.

19. Ronque ERV, Cyrino ES, Dórea VR, Serassuelo Júnior H, Galdi EHG, Arruda M. Prevalência de sobrepeso e obesidade em escolares de alto nível socioeconômico em Londrina, Paraná, Brasil. Rev Nutr. 2005; 18(6):709-17.

20. Barbetta PA. Estatística aplicada às Ciências Sociais. Florianópolis: UFSC; 2003. 
21. Ross WD, Marfell-Jones MJ. Kinanthropometry. In: McDougall JD, Wenger HA, Green HJ, editors. Physiological testing of the elite athlete. Ottawa: Mutual; 1982.

22. National Center for Health Statistics. Available from: <http://www.cdc.gov/growthcharts>.

23. National Center for Health Statistics. Epi InfoTM 6. Available from: <http://www. cdc.gov/epiinfo/ Epi6/Elln6.htm>.

24. Waterlow JC, Buzina R, Keller W, Lane JM, Nichaman MZ, Tanner JM. The presentation and use of height and weight data for comparing the nutritional status of groups of children under the age 10 years. Bull World Health Org. 1977; 55(4): 489-98.

25. Anjos LA. Índices antropométricos e estado nutricional de escolares de baixa renda de um município do estado do Rio de Janeiro (Brasil): um estudo piloto. Rev Saúde Pública. 1989; 23(3): 221-9.

26. Chiara V, Sichieri R, Martins PD. Sensibilidade e especificidade de classificação de sobrepeso em adolescentes, Rio de Janeiro. Rev Saúde Pública. 2003; 37(2):226-31.

27. Rolland-Cachera MF, Bellisle F, Deheeger $M$, Pequignot $F$, Sempe M. Influence of body fat distribution during childhood on body fat distribution in adulthood: a two-decade followup-study. Int J Obes. 1990; 14(6):473-81.
28. Gross R, Stange M, Solomons NW, Olterrsddorf U, Esquivel IR. The influence of economic deterioration in Brazil on the nutritional status of children in Rio de Janeiro, Brazil. Ecol Food Nutr. 1987; 19(2):265-79.

29. Victora CG, Barros FC, Vaughan JP. Epidemiologia da desigualdade: um estudo longitudinal de 6.000 crianças brasileiras. São Paulo: Hucitec; 1988.

30. Taddei JAAC. Desvios nutricionais em menores de cinco anos: evidências dos inquéritos antropométricos nacionais [tese de livre-docência]. São Paulo: Escola Paulista de Medicina; 2000.

31. Pelto GH, Urgello J, Allen LH, Chavez A, Martinez $H$, Meneses $L$, et al. Household size, food intake and anthropometric status of school-age children in a highland Mexican area. Soc Sci Med. 1991; 33(10):1135-40.

32. Guimarães LV, Latorre MRDO, Barros MBA. Fatores de risco para a ocorrência de déficit estatural em pré-escolares. Cad Saúde Pública. 1999; 15(3): 605-15.

33. Magalhães VC, Mendonça GAS. Prevalência de fatores associados a sobrepeso e obesidade em adolescentes de 15 a 19 anos das regiões Nordeste e Sudeste do Brasil, 1996 e 1997. Cad Saúde Pública. 2003; 19(Supl 1):129-39.

Recebido em: 31/5/2007

Versão reapresentada em: 24/4/2008

Aprovado em: 17/6/2008 